\title{
Vacuolar Protein Sorting: Two Different Functional States of the AAA-ATPase Vps4p
}

\author{
Claudia Hartmann $^{1,2}$, Mohamed Chami ${ }^{2}$, Ulrich Zachariae ${ }^{3}$, \\ Bert L. de Groot ${ }^{3}$, Andreas Engel ${ }^{2 \star}$ and Markus G. Grütter ${ }^{1 *}$
}

\author{
${ }^{1}$ Institute of Biochemistry, \\ University of Zürich, \\ Winterthurer Strasse 190, \\ 8057 Zürich, Switzerland \\ ${ }^{2}$ M.E. Institute for Structural \\ Biology, Biozentrum, \\ University of Basel, \\ 4056 Basel, Switzerland \\ ${ }^{3}$ Max Planck Institute for \\ Biophysical Chemistry, \\ 37077 Göttingen, Germany
}

Received 26 September 2007; received in revised form

19 December 2007;

accepted 4 January 2008

Available online

15 January 2008

Edited by W. Baumeister

\begin{abstract}
The vacuolar protein sorting (Vps) pathway, in which Vps4 class I AAAATPases play a central role, regulates growth factor receptors, immune response, and developmental signaling, and participates in tumor suppression, apoptosis, and retrovirus budding. We present the first atomic structure of the nucleotide-free yeast $\mathrm{His}_{6} \Delta \mathrm{NVps} 4 \mathrm{p}$ dimer and its AMPPNP (5'adenylyl- $\beta, \gamma$-imidodiphosphate)-bound tetradecamer, derived from a cryo electron microscopy map. Vps4p dimers form two distinct heptameric rings and accommodate AAA cassettes in a head-to-head-not in a head-to-tailfashion as in class II AAA-ATPases. Our model suggests a mechanism for disassembling ESCRT (endosomal sorting complex required for transport) complexes by movements of substrate-binding domains located at the periphery of the tetradecamer during ATP hydrolysis in one ring, followed by translocation through the central pore and ATP hydrolysis in the second ring.
\end{abstract}

(C) 2008 Elsevier Ltd. All rights reserved.

\section{Introduction}

The eukaryotic vacuolar protein sorting (Vps) pathway is important for the sorting of ubiquitinated cell surface receptors and lysosomal hydrolases from the endosome to the lysosome. ${ }^{1}$ The human Vps pathway participates in medically relevant processes such as tumor suppression, ${ }^{2}$ cell death, ${ }^{3}$ or budding of retroviruses such as HIV. ${ }^{4}$ In yeast, 18 different proteins are involved in Vps. ${ }^{5}$ Each yeast Vps protein has one or two human orthologues, ${ }^{5}$ suggesting that the yeast Vps pathway could serve as a model system for the more complicated human machinery. The highly conserved Vps pathway is a multistep process that requires the assembly and disassembly of a cascade of protein complexes called Vps27p complex, ESCRT (endosomal sorting complex

${ }^{*}$ Corresponding authors. E-mail addresses: andreas.engel@unibas.ch; gruetter@bioc.uzh.ch

Abbreviations used: Vps, vacuolar protein sorting; AMPPNP, 5 '-adenylyl- $\beta, \gamma$-imidodiphosphate; EM, electron microscopy; MD, molecular dynamics; EDTA, ethylenediaminetetraacetic acid. required for transport) I, ESCRT-II, and ESCRT-III. ${ }^{5}$ After ESCRT-III has performed its function, all ESCRT complexes are dissociated for the next transport cycle by the AAA-ATPase, Vps4p. Loss of Vps4p function in yeast leads to an aberrant multilamellar endosomal compartment and to missorting and secretion of vacuolar proteins. ${ }^{6}$

AAA-ATPase monomers contain either one (class I) or two (class II) nucleotide-binding domains per monomer, the so-called AAA-ATPase cassettes. ${ }^{7}$ These proteins function as oligomers, in most known cases forming hexameric rings. ${ }^{7}$ Vps4p is a class I AAA-ATPase that was reported to form dimers in the cytosol ${ }^{6,8}$ and decameric ${ }^{6}$ or dodecameric ${ }^{8}$ ATPbound complexes at the endosomal membrane, in contrast to the hexameric class I AAA-ATPase, $\mathrm{HslU}^{9}$ or FtsH. ${ }^{10} \mathrm{Vps} 4$ proteins can be divided into three domains: (i) the N-terminal domain, which contains a MIT-(N-terminal microtubule interacting and trafficking) domain with an 'up-and-down' three-helix bundle and a flexible long linker; ${ }^{11-14}$ (ii) the highly conserved large AAA-ATPase domain with a mixed $\alpha / \beta$ fold and the ATP-binding site; 8,15 and (iii) the structurally less well-conserved small AAA-ATPase domain with a four-helix bundle, an 
inserted $\beta$-domain, and a C-terminal helix. ${ }^{8,15}$ The $\beta$-domain seems to be specific for Vps4 proteins, ${ }^{8,15}$ while the C-terminal helix is also found in p97-D2. ${ }^{16}$

During the ESCRT disaggregation process, several components of the sorting machinery interact directly with Vps4p-some via its $\beta$-domain ${ }^{8,17-19}$ and some via its MIT-domain ${ }^{11,13,14,19-23}$ - with the latter being key to the recruitment of Vps4p to the endosome. ${ }^{6}$ The binding partners are bound with their highly conserved C-terminal helix to the MIT domain. ${ }^{13,14,21}$ In addition, mutations in the binding motifs of the MIT-domain enhance the interactions mediated by the $\beta$-domain and vice versa, suggesting a functional interaction of the MIT domain and the $\beta$-domain. ${ }^{20}$ Finally, based on a ring-like hexameric model of Vps4B using the structure of p97, the highly conserved pore residue tryptophan (W206) was mutated to alanine. This blocked HIV budding in vivo and indicated that substrates are translocated through the central pore. ${ }^{8}$

Here we present the first atomic structure of the nucleotide-free $\mathrm{His}_{6} \Delta \mathrm{NVps} 4 \mathrm{p}$ dimer from yeast at $3.35 \AA$ resolution and a model of its 5'-adenylyl$\beta, \gamma$-imidodiphosphate (AMPPNP)-bound tetradecamer, derived from an $18-\AA$ map obtained by cryo electron microscopy (EM). These structures show a head-to-head arrangement of AAA cassettes in the Vps4p dimer and in the heptameric double-ring structure comprising $14 \mathrm{Vps} 4 \mathrm{p}$ monomers, in contrast to the head-to-tail arrangement ${ }^{16,24-27}$ reported for class II AAA-ATPases. The head-to-head dimer of $\mathrm{His}_{6} \Delta \mathrm{NVps} 4 \mathrm{p}$ is confirmed to be structurally stable in molecular dynamics (MD) simulations. In addition, a point mutant at the dimer head-to-head interface prevented oligomerization. The two heptameric rings differ significantly in shape and in the diameter of their pores. These features lead to a model of the mechanism of ESCRT disassembly.

\section{Results}

\section{Structure of apo-His ${ }_{6} \Delta \mathrm{NVps} 4 p$}

We have cloned, expressed, purified, and crystallized the N-terminal-deleted, His-tagged, nucleotide-free Vps4p, called $\mathrm{His}_{6} \Delta \mathrm{NVps} 4 \mathrm{p}$, as described in Materials and Methods. The protein crystallized after 5 days in space group $P 6_{5} 22$, giving a resolution of $3.35 \AA$. The structure was solved by molecular replacement using the structure of $\mathrm{Vps} 4 \mathrm{~B}^{8}{ }^{8}$ revealing one apo-His ${ }_{6} \Delta \mathrm{NVps} 4 \mathrm{p}$ molecule in the asymmetric unit, resulting in a crystal packing with a dimer forming a helix around the z-axis. The protomers in the dimer are related by a crystallographic 2-fold (a) (b)

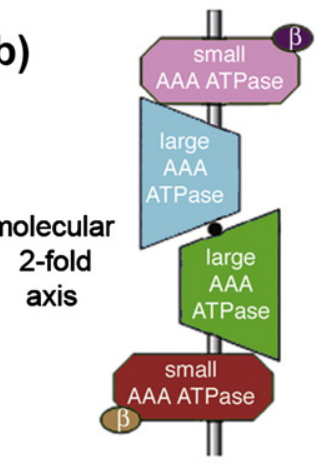

Vps4p molecular 7-fold axis

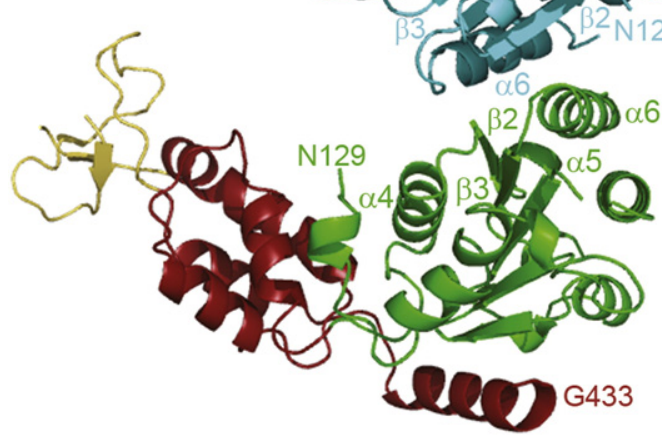

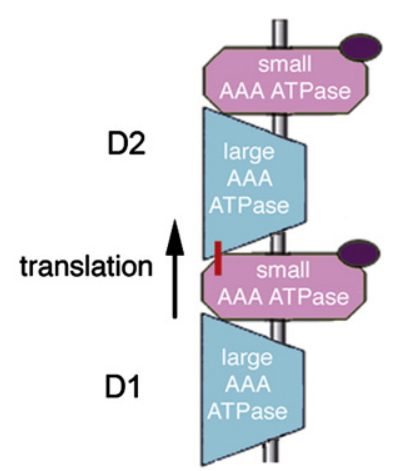

P97 molecular 6-fold axis

Fig. 1. The structure of the His ${ }_{6} \Delta$ NVps4p dimer. (a) Ribbon diagram of the His ${ }_{6} \Delta$ NVps $4 p$ dimer viewed along the 2-fold axis. The large AAA-ATPase domains are in green and cyan, the small AAA-ATPase domains are in light purple and red, and the $\beta$-domains are in dark purple and brown, respectively. The secondary structure elements at the dimer interface are the same for both protomers. (b) The top picture shows a schematic view of the domain arrangement of the class I AAAATPase $\mathrm{His}_{6} \Delta \mathrm{NVps} 4 \mathrm{p}$ dimer. The colors correspond to the ones in (a). The bottom picture illustrates the domain arrangement in the class II AAA-ATPase p97 for comparison. The linker between the two AAA cassettes in p97 is in red. 
Table 1. X-ray data and refinement statistics

\begin{tabular}{|c|c|}
\hline Data statistics & \\
\hline Wavelength $(\AA)$ & 1.0 \\
\hline Resolution $(\AA)$ & 3.35 \\
\hline$R_{\text {merge }}{ }^{\mathrm{a}}(3.53-3.35 \AA)$ & $5.7(44.0)$ \\
\hline Completeness (\%) (3.53-3.35 Å) & $99.4(100)$ \\
\hline$I / \sigma(I)(3.53-3.35 \AA)$ & $22.6(4.9)$ \\
\hline Multiplicity $(3.53-3.35 \AA$ A & $8.1(8.4)$ \\
\hline Unit cell $(\AA)$ & $109.116,176.632$ \\
\hline No. of independent reflections & 9446 \\
\hline Refinement statistics & \\
\hline Resolution $(\AA)$ & 3.35 \\
\hline$R_{\text {work }}{ }^{\mathrm{b}} / R_{\text {free }}{ }^{c}$ & $26.8 / 29.8$ \\
\hline r.m.s.d. & \\
\hline Bond lengths $(\AA)$ & 0.008 \\
\hline Bond angles $\left(^{\circ}\right)$ & 1.107 \\
\hline No. of atoms & \\
\hline Protein & 1837 \\
\hline $\begin{array}{l}\left.{ }^{\mathrm{a}} R_{\text {sym }}=\sum h k l|I(h k l)-\langle I(h k l)\rangle| /\right\rangle \\
\text { mean of the symmetry-equivaler } \\
\mathrm{b} R=\sum\left(\left|F_{\text {obs }}\right|-k\left|F_{\text {calc }}\right|\right) / \sum \mid F_{\mathrm{o}} \\
\mathrm{c} \text { Free } R \text { value obtained for a t } \\
\text { a randomly selected } 5 \% \text { subset } \\
\text { refinement. }\end{array}$ & $\begin{array}{l}\text { Ie }\langle I(h k l)\rangle \text { is the } \\
I(h k l) \text {. } \\
\text { ions consisting of } \\
\text { data not used in }\end{array}$ \\
\hline
\end{tabular}

symmetry (Fig. 1a and b, top). The current structure contains residues 129-204, 208-238, and 248-263, 268263, 268-298, together forming the large AAA-ATPase domain (Fig. 1a, green and blue), and residues 299-357 and 400-418, comprising the four-helix bundle of the small AAA-ATPase domain (Fig. 1a, light purple and red), with an inserted $\beta$-domain from residues 358-363 and 374-382, 384-399 (Fig. 1a, yellow and dark purple) and with the C-terminal helix containing residues 419432. Table 1 summarizes the data and refinement statistics. We also performed crystallization experiments of full-length $\mathrm{His}_{6} \mathrm{Vps} 4 \mathrm{p}$. Under a different condition, the crystals exhibiting the same crystal packing as truncated $\mathrm{His}_{6} \mathrm{Vps} 4 \mathrm{p}$ grew after 4 months and diffracted to $4 \AA$, but the N-terminal domain was not visible upon determination of electron density. Subsequent sequencing of the protein from dissolved crystals revealed that this part was missing due to proteolytic cleavage at ${ }^{123} \mathrm{I}$.

The two subunits of our $\mathrm{His}_{6} \Delta \mathrm{NVps} 4 \mathrm{p}$ dimer are related by a 2 -fold axis, with the interface mediated by the large AAA-ATPase domains (Fig. 1a and b, top, green and blue) of both monomers, arranging the AAA cassettes in a head-to-head configuration, as also observed for the full-length Vps4p where the $\mathrm{N}$-terminal part with its His-tag was missing due to proteolytic cleavage. This is in contrast to its most similar structure of the class II AAA-ATPase p97, where cassettes D1 and D2 are arranged head-totail $^{16,24,25}$ (Fig. 1b, bottom).

In Vps4p, the contact between the protomers is mediated by residues of helices $\alpha 4, \alpha 5$ and $\alpha 6$, the $\beta$-strand $\beta 2$, and the three loops between $\alpha 4$ and $\beta 2$, $\alpha 5$ and $\alpha 6, \alpha 6$ and $\beta 3$ of both subunits (Fig. 1a). The interface of $\sim 635 \AA^{2}$ represents $4.0 \%$ of the $\mathrm{His}_{6-}$ $\Delta N V p s 4 p$ surface area, with a shape complementarity of 0.551 and a $P$ value of 0.399 , analyzed with the CCP4 package ${ }^{28}$ and the PISA server. ${ }^{29}$ This interface area includes the disordered solventexposed side chains of our atomic model (Supplementary Table 1); their exclusion decreases the interface to $\sim 454 \AA^{2}$. Mainly, the residues T193, F195, L213, L217, and M220 of both protomers contribute with their hydrophobic side chains to the formation of the dimer. In addition, the side chains of residues N224 and Q216 of subunits A and B form six hydrogen bonds to the main chains of F194 and L213 of subunits B and A, respectively (Fig. 2).

\section{Biophysical and biochemical analyses of Vps4p oligomerization}

Analytical ultracentrifugation analysis was used to confirm that $\mathrm{His}_{6} \Delta \mathrm{NVps} 4 \mathrm{p}$ also exists as a higher oligomeric assembly in the presence of AMPPNP or ADP vanadate. The results demonstrated that AMPPNP or ADP vanadate induces an assembly of higher oligomers, irrespective of the presence of ethylenediaminetetraacetic acid (EDTA) (data not shown). Analytical ultracentrifugation and crosslinking experiments yielding species in the range of dodecamers/tetradecamers and huge aggregates at nonphysiological concentrations $(>7 \mathrm{mg} / \mathrm{ml}$ ) (data not shown) agree with earlier gel-filtration experiments at higher concentrations $(\sim 5 \mathrm{mg} / \mathrm{ml})$ in the
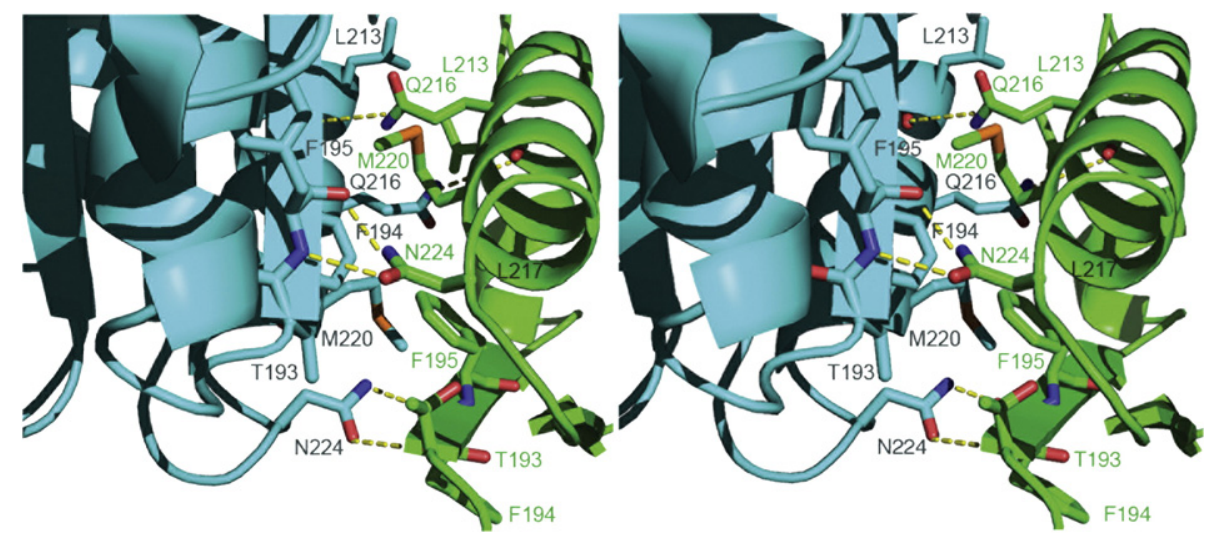

Fig. 2. Interface formed by the protomers in the $\mathrm{His}_{6} \Delta \mathrm{NVps} 4 \mathrm{p}$ dimer. The residues contributing with their hydrophobic side chain to the interaction surface are displayed, and the six hydrogen bonds involved in this interaction are shown in yellow. 
presence of nucleotide. ${ }^{8}$ Cross-linking at lower concentrations $(\sim 0.2 \mathrm{mg} / \mathrm{ml})$, followed by SDSPAGE analysis, revealed a band above $550 \mathrm{kDa}$, as well as smaller species (data not shown), as also observed by Babst et al. ${ }^{6}$ EM of negatively stained cross-linked preparations showed heterogeneous particles with a size range of about $20 \mathrm{~nm}$ and some smaller particles (data not shown). However, all our attempts to produce such higher oligomers with the full-length $\mathrm{His}_{6} \mathrm{Vps} 4 \mathrm{p}$ were not successful.

\section{Cryo-EM}

To structurally analyze the functional Vps4p complex, cryo-EM of $\mathrm{His}_{6} \Delta \mathrm{NVps} 4 \mathrm{p}$ was performed in the presence of AMPPNP. Micrographs recorded at low dose reveal top and side views of ring-shaped particles, as well as filaments (Fig. 3a). Most of the filaments dissociated upon addition of $5 \mathrm{mM}$ EDTA and more single particles were observed (Fig. 3b), since EDTA does not inhibit AMPPNP binding as demonstrated for other AAA-ATPases. ${ }^{9}$ Cryo-EM of nucleotide-free $\mathrm{His}_{6} \Delta \mathrm{NVps} 4 \mathrm{p}$ preparation confirmed the expected lack of large ring-shaped oligomers (data not shown).

A total of 6904 projections comprising all possible projection angles were manually selected, submitted to a reference-free alignment without imposed symmetry, ${ }^{30}$ and classified. This was executed with XMIPP ${ }^{31}$ which centers the projections and classifies them by their angular spectra. Of the 49 classes, 5 exhibited a single peak at the seventh angular harmonic. These 5 classes representing the top views of the Vps4p complex comprised 631 particles. A total of 731 projections populating 27 classes showed a prominent 2-fold symmetry corresponding to the side views. The averages of all these classes were subsequently calculated using EMAN. ${ }^{32}$ A characteristic average of side views and one of top views, and the respective rotational power spectra are shown in Fig. 3c and d. Accordingly, the Vps4p complex consists of two stacked rings, each comprising seven subunits.

However, classes with 6- or 8-fold symmetry were also found, but they represented a smaller fraction of projections with populations of 243 and 239, respectively (Supplementary Fig. 1a and b, top). Visual inspection of averages calculated from these classes with EMAN ${ }^{32}$ showed projections of slightly tilted particles still exhibiting seven distinct subunits (Supplementary Fig. 1a and b, bottom). To further confirm the heptameric stoichiometry of the complex, all the manually selected 3740 top views were submitted to a reference-free alignment using EMAN ${ }^{32}$ to yield 37 class averages, of which all classes revealing distinct subunits had 7 of them, and comprised a total of 1203 projections (Supplementary Fig. 2).

A 3D reconstruction was achieved with 2473 selected projections of the AMPPNP-complexed $\mathrm{His}_{6} \Delta \mathrm{NVps} 4 \mathrm{p}$ that were structurally well preserved, starting from a simple model of a heptameric double ring, as detailed in Materials and Methods. When all 6904 were used to obtain the 3D
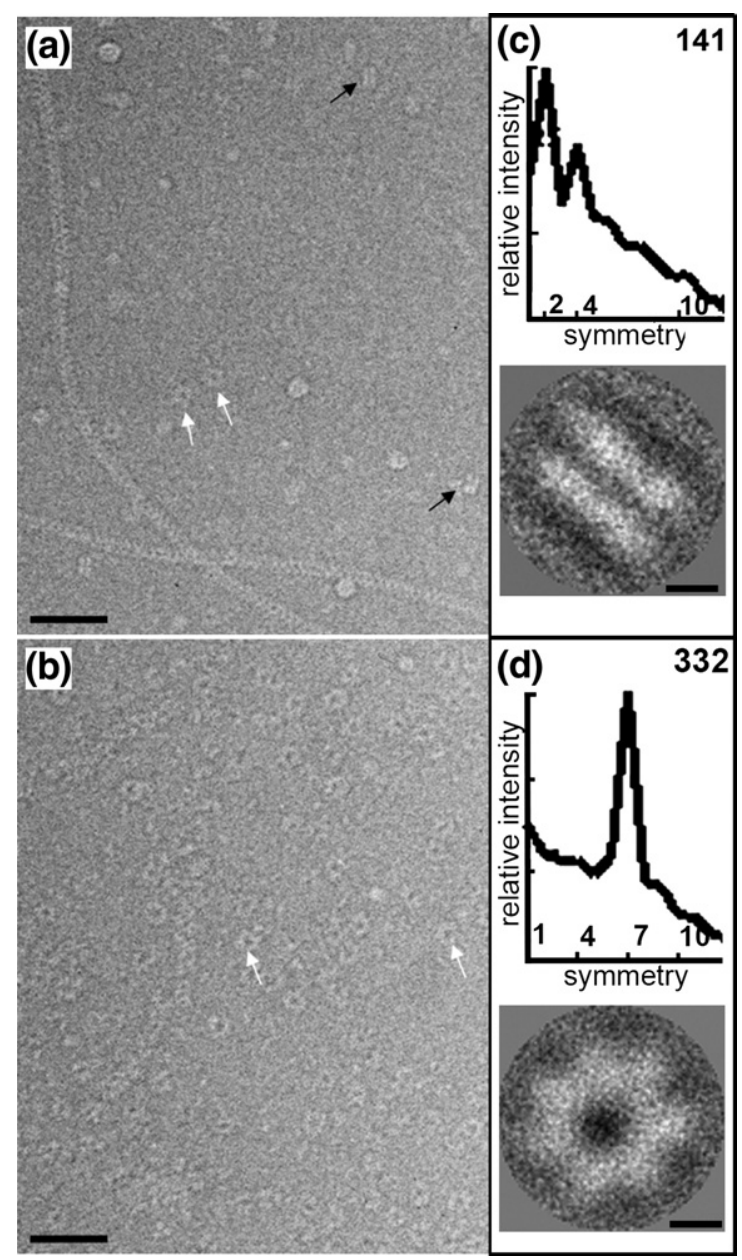

Fig. 3. Cryo-EM of $\mathrm{His}_{6} \Delta \mathrm{NVps} 4 \mathrm{p}$ complexed with AMPPNP. (a and b) Micrographs of $\mathrm{His}_{6} \Delta \mathrm{NVps} 4 \mathrm{p}$ complexed with AMPPNP. The top and side views are marked by white and black arrows, respectively. The scale bar represents $500 \AA$. (a) The sample region without EDTA shows filaments and side views. (b) The sample region with EDTA reveals top views. (c and d) Rotational power spectra (with symmetry on the $x$-axis and with relative intensity on the $y$-axis) and their corresponding referencefree class averages without the imposed symmetry of side (c) and top views (d) are displayed, with the scale bar representing $50 \AA$. The number of particles per class is printed at the right top edge of each power spectrum.

reconstruction, a resolution of $30 \AA$ was achieved. Refining the map with either 6904 or 2473 projections yielded maps with the same morphology, but only the reconstruction from the selected projections produced a resolution of $18 \AA$ (Supplementary Fig. 3 ), indicating distinct conformational variability. ${ }^{33}$

This map was contoured to comprise a mass of $512 \mathrm{kDa}$ (corresponding to a $\mathrm{His}_{6} \Delta \mathrm{NVps} 4 \mathrm{p}$ tetradecamer) (Fig. 4a1) to yield an outer diameter of the complex of $150 \AA$ and a height of $80 \AA$. The two heptameric ATPase rings differ significantly in size and in shape, having pore diameters of $40 \AA$ and $55 \AA$, respectively (Fig. 4a1), as illustrated by contour plots of the sections through the median plane of the two rings (Fig. $4 \mathrm{a} 2$ and a3). 
(a)
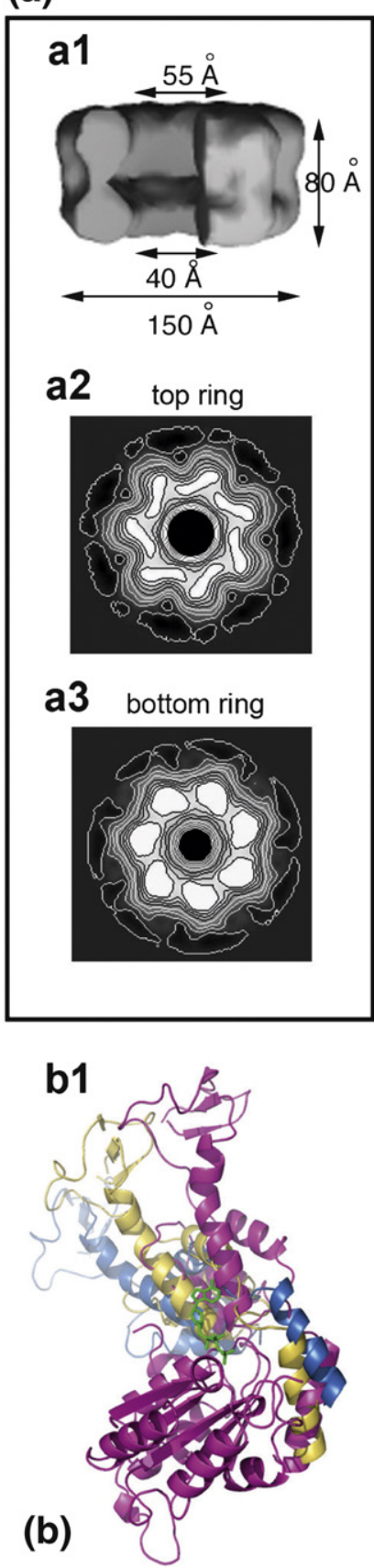

b2
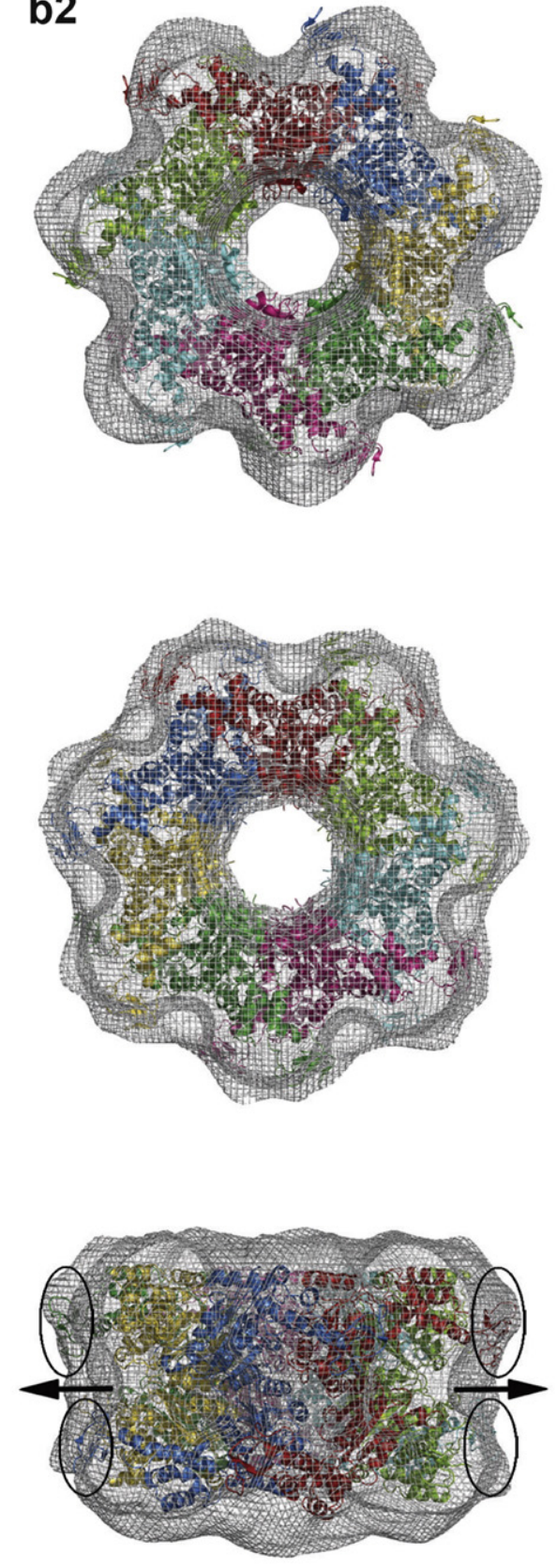

(c)

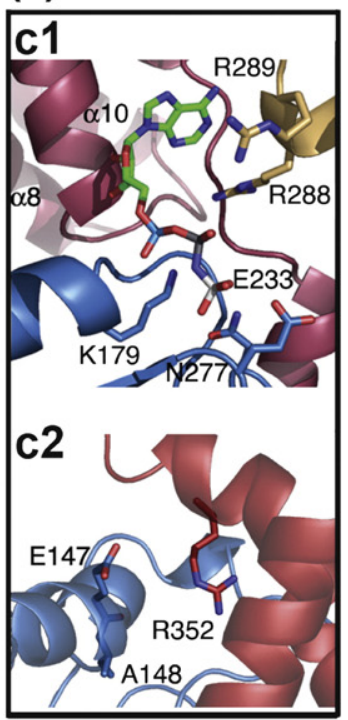

(d)

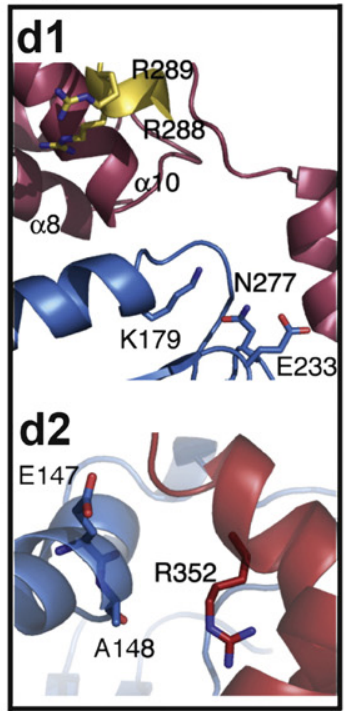

Fig. 4. Structure of the $\mathrm{His}_{6} \Delta \mathrm{NVps} 4 \mathrm{p}$ tetradecamer. (a1) A cut through the $3 \mathrm{D}$ reconstruction of $\mathrm{His}_{6} \Delta \mathrm{NVps} 4 \mathrm{p}$ from cryo-EM projections shows the difference between the top ring and the bottom ring. A contour plot of the sections through the median plane perpendicular to the $z$-axis of the top AAA-ATPase ring (a2) and the bottom AAA-ATPase ring (a3) of $\mathrm{His}_{6} \Delta$ NVps4p is shown for comparison. (b1) The apo- and AMPPNP-complexed protomers as fitted into the EM map, and the apo-protomer of the dimer of $\mathrm{His}_{6} \Delta \mathrm{NVps} 4 \mathrm{p}$ in blue, gold, and pink, respectively, are superimposed on their large AAA-ATPase domains. The small AAA-ATPase domains show different orientations relative to the large AAA-ATPase domains. (b2) Different views of the reconstruction of $\mathrm{His}_{6} \Delta \mathrm{NVps} 4 \mathrm{p}$ are displayed as a mesh with a contour level of $2.5 \sigma$, with the tetradecamer model fitted inside. Shown are the AMPPNP-complexed AAA-ATPase ring, the apo-ATPase ring, and a side of the double-ring 3D reconstruction (from top to bottom). The positions of the deleted N-domains and $\beta$-domains are highlighted in the side view of the tetradecamer by arrows and ellipses, respectively. (c1-d2) Features of the $\mathrm{His}_{6} \Delta \mathrm{NVps} 4 \mathrm{p}$ tetradecamer structure as fitted into the EM map. (c1) Plot of the nucleotide-binding site with the catalytic residues of the AMPPNP-complexed AAA-ATPase ring with the large, small, and neighboring large AAAATPase domains in blue, pink, and gold, respectively. (c2) Diagram of the contact region between the small AAA-ATPase domain (in pink) and the neighboring large AAA-ATPase domain (in blue) for the AMPPNP-complexed AAA-ATPase ring. (d1) A close-up view of the nucleotide-binding site of the nucleotide-free AAA-ATPase ring with the large, small, and neighboring large AAA-ATPase domains in blue, pink, and gold, respectively. This reveals the closure of the ATP-binding site in the latter. (d2) A close-up view of the contact region between the small AAA-ATPase domain (in pink) and the neighboring large AAA-ATPase domain (in blue) for the apo-ATPase ring. 
As detailed in Materials and Methods, we fitted the atomic model of the $\mathrm{His}_{6} \Delta \mathrm{NVps} 4 \mathrm{p}$ dimer into the cryo-EM map, with the large AAA-ATPase domain dimer and the two small AAA-ATPase domains acting as three rigid bodies connected by hinge regions between the large and small AAAATPase domains in such a manner that no clashes between $C \alpha$ positions occurred when applying a 7fold symmetry (Fig. 4b2). In addition, we considered that the active site is preserved and that the conserved residue R352 of the small AAAATPase domain interacts with the neighboring large AAA-ATPase domain, such as the equivalent arginines of the D1 and D2 domains in p97 $16,24,25$ (Fig. 4c2 and d2). The nonfunctional point mutant Vps4p R352A corroborates this requirement. ${ }^{8}$ Only a small portion of the model corresponding to the loops delineating the pore does not fit the EM map, while some unoccupied space at the periphery of the EM map coincides with the disordered residues $364-373$ in the atomic $\mathrm{His}_{6} \Delta \mathrm{NVps} 4 \mathrm{p}$ model.

A comparison of the active sites of both rings of our model of the tetradecameric $\mathrm{His}_{6} \Delta \mathrm{NVps} 4 \mathrm{p}$ with p97 showed that AMPPNP fits well into the ATP-binding pocket of the subunits of the more open top ring (Fig. 4c1). Walker A (K179), Walker $\mathrm{B}$ (E233), and SRH (second region of homology; N277) of the large AAA-ATPase domain (blue), and helix $\alpha 10$ of the small AAA-ATPase domain (purple), as well as the SRH of the neighboring subunit with its arginine fingers (R288 and R289; gold), surround the nucleotide-binding site (Fig. $4 \mathrm{c} 1)$. The binding pocket of the subunits of the more closed bottom ring has a different conformation and is tighter, since the adenosinebinding site is blocked by the SRH region of the adjacent subunit (Fig. 4d1). In our fit, the orientations of large and small AAA-ATPase domains of both rings differ from each other and from the protomer in the apo dimer (Fig. 4b1, blue, gold and pink). We also fitted the apo- and nucleotide-complexed heptameric rings as headto-tail and tail-to-tail arrangements into the EM map (with correlation coefficients of $\sim 0.8$ determined with Situs ${ }^{34}$ ), and we found that the interface between the two rings decreased significantly from $635 \AA^{2}$ to $290 \AA^{2}$ and $310 \AA^{2}$ per dimer, respectively.

\section{Stability of different dimer models by MD simulations}

Because of the limited resolution of our 3D map from cryo-EM, the correlation coefficients by Situs ${ }^{34}$ did not allow the three possible arrangements of the Vps4p subunits in the heptameric complex to be discerned. Nevertheless, the interfaces of the different conformations varied greatly, favoring the head-to-head conformation found in the 3D crystal.

To further compare these interfaces, MD simulations were performed for all three possibilities over

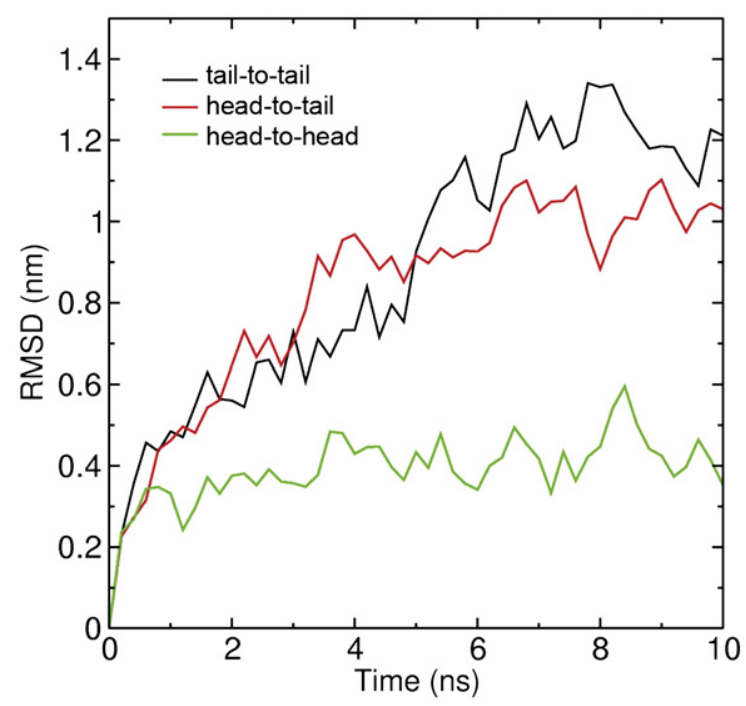

Fig. 5. Backbone r.m.s.d. during 10-ns MD simulations of dimers contacting at different surface areas. The simulations of the dimer in the head-to-head, tail-to-tail, and head-to-tail orientations are displayed in green, red, and black, respectively. The tail-to-tail and head-to-tail arrangements show large structural changes and the beginning of dissociative behavior, while the head-tohead arrangement is structurally stable in the simulations.

$10 \mathrm{~ns}$, as described in Materials and Methods. As displayed in Fig. 5, only the dimer contacting in the head-to-head orientation proved to be structurally stable in the simulations (green trace). Both the head-to-tail-oriented dimer (red trace) and the tailto-tail-oriented dimer (black trace) show large structural changes and the beginning of a dissociative behavior. This result demonstrates the structural stability of the head-to-head configuration of the Vps4p dimer and supports the packing models of the dimer and the tetradecamer of Vps4p displayed in Figs. 1a and 4b2.

\section{Oligomerization of wild-type and mutated Vps4p}

In the absence of nucleotide, gel filtration of $\mathrm{His}_{6} \mathrm{Vps} 4 \mathrm{p}$ at a concentration of $5 \mathrm{mg} / \mathrm{ml}$ revealed a single dimer peak, while $\mathrm{His}_{6} \Delta \mathrm{NV}$ ps4p showed a dimer and a higher-molecular-weight peak (Fig. 6).

To verify the physiological relevance of the headto-head arrangement, we mutated the interface residue Q216 to Ala and analyzed its oligomeric state by gel filtration. His ${ }_{6} \Delta \mathrm{NVps} 4 \mathrm{p}$ Q216A eluted as a monomer (Fig. 6). In addition, in the presence of AMPPNP with and without EDTA, $\mathrm{His}_{6} \Delta \mathrm{NV}$ ps4p Q216A showed a lack of tetradecamers and filaments on cryo-EM examinations.

\section{Discussion}

Here we present the first structure of a type I AAAATPase in two functional states as a nucleotide-free 


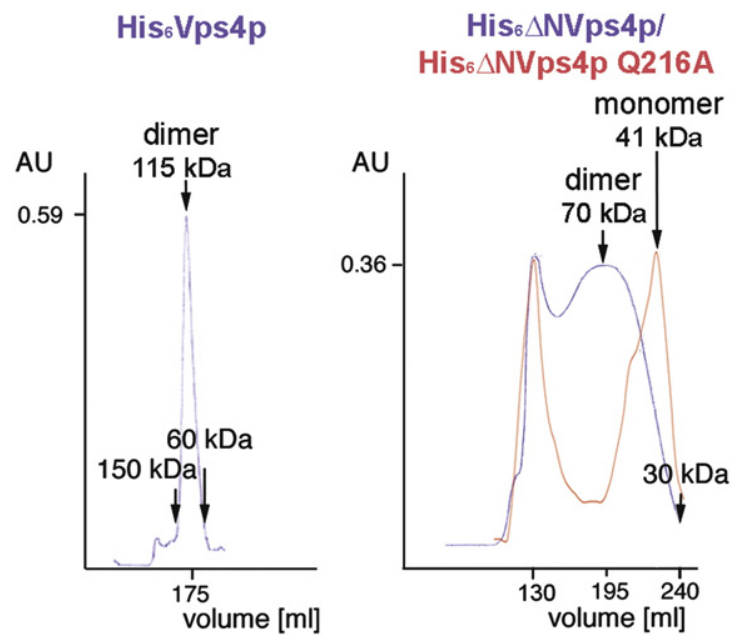

Fig. 6. ATPase activity and gel filtration of $\mathrm{His}_{6} \mathrm{Vps} 4 \mathrm{p}$ and $\mathrm{His}_{6} \Delta \mathrm{NVps} 4 \mathrm{p}$. The gel-filtration profiles of $\mathrm{His}_{6} \mathrm{Vps} 4 \mathrm{p}$ and $\mathrm{His}_{6} \Delta \mathrm{NVps} 4 \mathrm{p} / \mathrm{His}_{6} \Delta \mathrm{NVps} 4 \mathrm{p}$ Q216A are compared and illustrate the inability of the Q216A mutant to form dimers.

dimer and as an AMPPNP-complexed tetradecamer. The AAA-ATPase cassettes in the Vps4p dimer and between the asymmetric heptameric rings are arranged in a head-to-head configuration. In contrast, the two covalently linked AAA-ATPase cassettes of the closest relative of Vps4p, the class II AAA-ATPase p97, are arranged head-to-tail and assemble into two asymmetric hexameric AAAATPase rings. ${ }^{16,24,25}$

\section{Comparison between Vps4 proteins}

At concentrations of $5 \mathrm{mg} / \mathrm{ml}$, both the full-length $\mathrm{His}_{6} \mathrm{Vps} 4 \mathrm{p}$ and the truncated $\mathrm{His}_{6} \Delta \mathrm{NVps} 4 \mathrm{p}$ eluted as stable dimers in gel-filtration experiments (Fig. 6), in agreement with earlier studies.6,8 The single-point mutant Q216A in $\mathrm{His}_{6} \Delta \mathrm{NVps} 4 \mathrm{p}$ disrupted the dimer formation, as shown in Fig. 6, demonstrating the relevance of the head-to-head interface observed in our crystal packing arrangement (Fig. 1). In comparison, Vps4B eluted at a monomer/dimer equilibrium at similar concentrations ${ }^{8}$ and is supposed to form a heterodimeric complex with Vps4A. ${ }^{35}$

Most recently, the monomeric structures of apo- and ADP-complexed Vps4p structures were published. ${ }^{15}$ In the nucleotide-free monomeric Vps4B ATPase domain structure ${ }^{8}$ and in the monomeric ADPcomplexed Vps4p structure, ${ }^{15}$ residues 124-126 form an antiparallel $\beta^{\prime}$-stand, while these residues in the monomeric apo-Vps4p structure ${ }^{15}$ are disordered. In fact, if the side chains of residues 124-126 in our complex would adopt an equivalent conformation as in Vps4B and the ADP-complexed Vps4p monomers, they would clash with the residues from helix $\alpha 6$ of the second protomer in our dimeric complex and thus sterically hinder the dimer formation between two Vps4p protomers. This $\beta^{\prime}$-strand is present only in the ADP-complexed Vps4p ${ }^{15}$ and in apo-Vps4B, which does not dimerize but forms heterodimers with
Vps4A, but neither in our $\mathrm{His}_{6} \Delta \mathrm{NVps} 4 \mathrm{p}$ structure nor in the monomeric apo-Vps4p structure. ${ }^{15}$ Therefore, this element is unlikely to be very stable and may adopt another conformation during dimer formation. The r.m.s.d. between the $\mathrm{C} \alpha$ atoms of the human and yeast Vps4 monomers is $1.3 \AA$ (the large and small AAA-ATPase domains of Vps4p were superimposed separately on the human Vps4B structure).

\section{Oligomerization of Vps4p}

Over 6900 projections of vitrified Vps4p complexes were selected to calculate the 3D map from projections of vitrified samples recorded by a highresolution cryo-electron microscope. Top-view class averages from reference-free aligned particle populations revealed an unambiguous 7-fold symmetry, while side-view class averages suggested the existence of two stacked unequal rings (Fig. $3 c$ and $d$ ). This is in contrast to the observation of a decameric ${ }^{6}$ or a dodecameric ${ }^{8} \mathrm{Vps} 4 \mathrm{p}$ in the presence of ATP. Indeed, most of the AAA-ATPases are hexameric, although for $\mathrm{ClpB}^{27,36}$ and HslU, ${ }^{9,37}$ both hexameric and heptameric rings have been observed. The oligomerization state appears to vary depending on the conditions or protein species, and does not seem to be critical to the function of the respective AAA-ATPase.

The deletion of substrate-binding domains in AAA-ATPases has no influence on the number of subunits in the ATP-complexed oligomer because the interactions between the subunits in the AAAATPase rings are mediated by the AAA cassettes and not by their substrate-binding domains. The latter are located distal from the ATPase rings as observed in p97, ${ }^{16,24,25} \mathrm{ClpB}^{27} \mathrm{HslU}^{9}{ }^{9}$ and $\mathrm{ARC},{ }^{38}$ similar to the model of the tetradecameric Vps4p (Fig. 4b2).

There is recent evidence that the MIT and $\beta$-domains interact functionally. ${ }^{20}$ Fitting our $\mathrm{His}_{6} \Delta \mathrm{NVps} 4 \mathrm{p}$ structure into the $18-\AA$ envelope of the $3 \mathrm{D}$ map suggests that the MIT- and $\beta$-domains are located at the periphery of the tetradecamer in rather close proximity (Fig. 4b2, bottom), in agreement with the findings of Vajjhala et al. ${ }^{20}$ Accordingly, the N-terminal deletion mutant $\mathrm{His}_{6} \Delta \mathrm{NVps} 4 \mathrm{p}$, in which the functional interaction between the MITdomain and the $\beta$-domain does not exist, assembled readily into a tetradecamer in vitro at a concentration of $2.5 \mathrm{mg} / \mathrm{ml}$ in the presence of ATP, while fulllength $\mathrm{His}_{6} \mathrm{Vps} 4 \mathrm{p}$ did not. Therefore, the functional interaction between the two domains could favor the dimeric form in the cytosol.

\section{Domain arrangement in Vps4p dimers and tetradecamers}

In our study, Vps4p forms dimers in the absence of nucleotide and asymmetric heptametic double rings comprising 14 protomers in the presence of AMPPNP. The head-to-head packing of AAAATPase cassettes in the dimer was found to involve an interface of $\sim 635 \AA^{2}$ comprising six hydrogen 
bonds and a number of hydrophobic interactions. The potential crystallographic dimer interaction surface in the most recently published Vps4p structure was different from our dimer interface and was identified as a packing artifact by point mutants (M330D/L407D), which had no effect on oligomerization in gel-filtration experiments. ${ }^{15}$ Fitting our dimer into the 3D map determined by cryoEM was feasible but required rotations of the small AAA domains with respect to the large AAA domains packed in the head-to-head configuration (Fig. 4b1).

The model of the tetradecameric Vps4p oligomer is supported by our data and a number of previous observations:

(1) The mutant Q216A of the interface residue involved in two hydrogen bonds in the headto-head arrangement of $\mathrm{His}_{6} \Delta \mathrm{NVps} 4 \mathrm{p}$, which resulted in a monomeric form (Fig. 6);

(2) The inability of this mutant to assemble into ring-shaped complexes in the presence of AMPPNP;

(3) The comparison of the head-to-head arrangement with the head-to-tail or tail-to-tail arrangement of the dimer, which demonstrated a decrease in the interface from $635 \AA^{2}$ to $310 \AA^{2}$ and $290 \AA^{2}$, respectively;

(4) MD calculations, which showed that the headto-head arrangement is structurally more stable than the other arrangements (Fig. 5);

(5) The replacement of surface residues at opposite sites in the C-terminal helix of Vps4p, namely, E419/L423, E426/R430, and Q427/ D431, by alanine, which had no influence on the oligomerization in vitro;

(6) The opposite handedness of the $\mathrm{His}_{6} \Delta \mathrm{NVps} 4 \mathrm{p}$ rings revealed by the respective sections in Fig. $4 \mathrm{a} 2$ and a3, which would be the same in a head-to-tail arrangement suggested by the p97 homolog. ${ }^{39}$

Taken together, the quarternary structural arrangement of AAA-ATPase domains we observed may be a characteristic feature of double-ring class I AAA-ATPases.

\section{Conformational changes during ATP hydrolysis}

In our model of the Vps4p tetradecamer, one AAAATPase ring is complexed with AMPPNP, while the other exhibits a different conformation and is nucleotide-free. Figure $4 \mathrm{a} 2$ and a3 demonstrates the difference between the two Vps4p rings. The interface mutant Q216A abolished the formation of both dimer and tetradecamer, suggesting the contact region of the large AAA-ATPase domains of Vps4p in the dimer and tetradecamer to be the same. During ATP hydrolysis, the main movements are performed by the large AAA-ATPase domains.9,24 In our structure, movements of the large AAA-ATPase domains of one ring may thus influence the position of the protomers in the other ring during ATPase cycles. Further structural and biochemical studies are necessary to confirm this. The asymmetry of the two AAA-ATPase rings has also been observed in p97 with six passive ADP-complexed protomers in the small ring, stabilizing the hexamer, while the active protomers form the large ring and run through the ATPase cycle. ${ }^{24,39}$ In our structure, both rings, one after the other, are able to hydrolyze ATP. The binding of AMPPNP in one ring appears to induce negative cooperativity and to inhibit the binding of AMPPNP in the other ring. Another explanation could be the flip-flop mechanism proposed by Babst et al., who suggest noncooperativity of the nucleotide binding of Vps4p subunits. ${ }^{6}$

\section{A model of the disaggregation of ESCRT complexes by Vps4p}

Taking into account the current knowledge on the structure and function of AAA-ATPases and the results presented here, it is tempting to speculate how the Vps4p may work to disassemble the ESCRT complexes after having performed their sorting function. ${ }^{6}$ We propose a four-step process (Fig. 7).

At concentrations of $0.2-0.3 \mu \mathrm{M}$, which correspond to its concentration in the yeast cytosol, Vps4p exists as a dimer. ${ }^{6}$ In the first step, Vps4p dimers are recruited to the endosomal membrane by the binding of the MIT domains to the ESCRT-III protein Vps20p and the C-terminal helix of Vps2p in an ATPhydrolysis-dependent manner. ${ }^{14,20,22}$ This process is facilitated by the ATP-independent interaction of the MIT-domain with the C-terminal helix of Vps46p, ${ }^{14,19,20}$ which is required for efficient membrane association of Vps4p. ${ }^{19}$ ATP-bound Vps4p may then diffuse laterally to assemble into the ringshaped active Vps4p complex. This process is probably similar in mammalian cells, where Vps4 proteins bind to the charged multivesicular body proteins class of ESCRT-III proteins via the MITdomain. ${ }^{11,13,21}$ The binding of MIT-domain with ESCRT-III proteins may not only foster oligomerization of the Vps4p dimers but also make the $\beta$-domain available for substrate binding. ${ }^{19}$ In the second step, the latter binds Vta1p, which enhanced the ATPase activity of Vps4p by sixfold to eightfold and was suggested to stabilize the large Vps4p oligomer and to bind in addition to Vps46p. ${ }^{17-19}$ In contrast to the model of Stuchell-Brereton et al., we propose that, during ATP hydrolysis, the alternating interactions of the MIT domains with ESCRT-III proteins may arrange the ring-shaped Vps4p oligomer with its pore axis parallel with the membrane, concomitant with the Vta1p-induced increase in ATPase activity and stabilization of the tetradecamer. ${ }^{13}$ This is supported by our model of the active Vps4p tetradecamer, where the MIT-domain is located at the equatorial periphery. In the third step, the ESCRT-III complexes are disassembled into smaller units by translating a small conformational change in the ATP-binding cleft into a large movement of the substrate-binding domains at the equatorial 


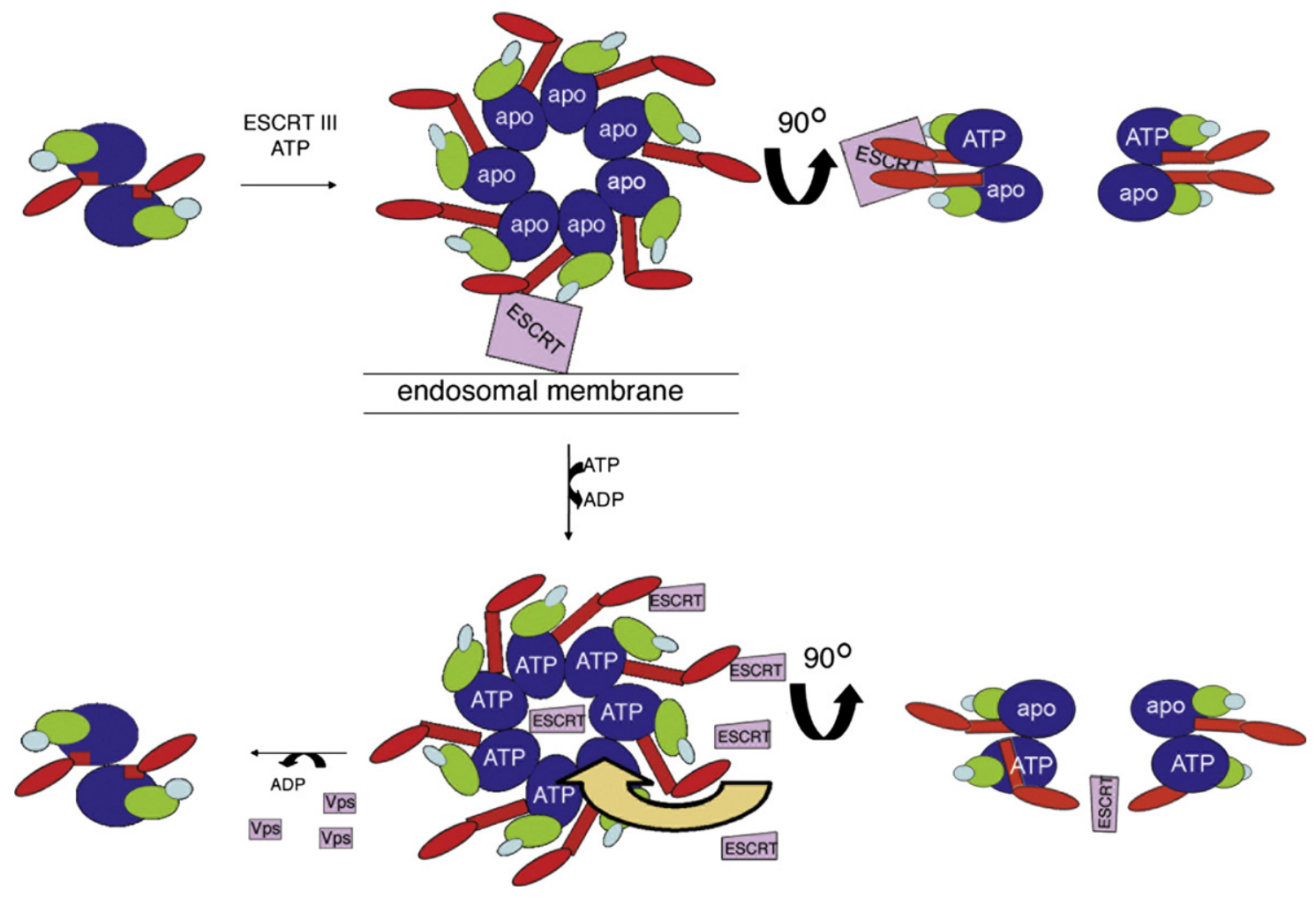

Fig. 7. A model for the disaggregation mechanism of Vps4p. In the cytosol, Vps4p is a dimer. The large AAA-ATPase domains are in blue, the small AAA-ATPase domains are in green, the $\beta$-domain is in light blue, and the MIT domain is represented by red ellipses. The linker between the MIT domain and the AAA-ATPase cassette is presented as a red rectangle. At the endosomal membrane, Vps4p is a tetradecamer. In this schematic picture, the tetradecamer is symbolized by a top view of one ring with its 7 -fold symmetry and a $90^{\circ}$ rotated slice through the heptameric double-ring structure. The ESCRT complexes are disassembled into smaller units by movements of the substrate-binding domains at the periphery of the tetradecamer during ATP hydrolysis in one ring and are then further disassembled into single proteins by translocation through the central pore during ATP hydrolysis in the other ring. Vps4p returns to the dimeric pool. The ESCRT complexes, smaller units of the ESCRT complexes, and single Vps proteins are shown as pink rectangles of different sizes.

periphery of the Vps4p tetradecamer during an ATPase cycle in one ring, similar to $\mathrm{ClpB}^{27}$ In the fourth step, the smaller units are transported by the flexible N-domains like an excavator to the pore of the larger of the two AAA-ATPase rings. Smaller ESCRT complexes are then further disassembled during ATP hydrolysis into single proteins and translocated through the central pore, whose critical role has been identified by mutations of putative pore residues. ${ }^{8}$ With its $40-55 \AA$ diameter, the pore is wide enough to translocate folded or partially folded proteins, in contrast to the small pore of $\mathrm{ClpB}^{27}$ and that of ATP-dependent proteases. ${ }^{9,10}$ Instead of disassembling into dimers after one round of disaggregation of ESCRT proteins, Vps4p tetradecamer may roll like a barrel along the membrane, remaining membrane-associated via its MIT-domains and binding to the farther substrates via its $\beta$-domains. Single ESCRT proteins return to the cytosol to start a new sorting cycle. Finally, upon consumption of the local ATP and the disappearance of the ESCRT complexes at the endosomal membrane, the Vps4p tetradecamer is destabilized and Vps4p returns to the dimeric pool in the cytosol. According to this model, both sides of the pore are accessible, and once the tetradecamer is assembled, it can disaggregate many ESCRT complexes before disassembling into dimers.

\section{Materials and Methods}

\section{Cloning, expression, and crystallization}

Full-length Vps4p (residues 2-437) and $\Delta N V p s 4 p$ (residues 124-437) amplified from the genomic DNA of baker's yeast were cloned between the BamHI and XhoI sites of the pet $20 \mathrm{~b}(+)$ vector with an N-terminal $\mathrm{His}_{6}{ }^{-}$ tag and a thrombin cleavage site for expression in Escherichia coli BL21(DE3) codon(+), called $\mathrm{His}_{6} \mathrm{Vps}_{4} \mathrm{p}$ and $\mathrm{His}_{6} \Delta \mathrm{NVps} 4 \mathrm{p}$, respectively. In addition, a point mutant Q216A was generated in the His-tagged $\Delta N V p s 4 p$, called $\mathrm{His}_{6} \Delta \mathrm{NVps} 4 \mathrm{p}$ Q216A, in a two-step PCR amplification protocol in which a pair of overlapping primers containing the mutation is used. Cells for all constructs were grown at $30{ }^{\circ} \mathrm{C}$ in LB medium to mid-log phase and induced by adding $0.05 \mathrm{mM}$ IPTG for overnight expression at $25{ }^{\circ} \mathrm{C}$. Cells were lysed and sonicated in the presence of the protease inhibitor cocktail Complete (EDTA-free; Roche), $20 \mathrm{mg}$ of lysozyme, and $1 \mathrm{mg}$ DNaseA/l culture. The lysates of both constructs were clarified by ultracentrifugation and applied to a 6-ml Ni-chelating column (Amersham Biosciences). For 
$\mathrm{His}_{6} \Delta \mathrm{NVps} 4 \mathrm{p}\left(\mathrm{His}_{6} \Delta \mathrm{NVps} 4 \mathrm{p}\right.$ Q216A) purification, NiNTA was equilibrated with $20 \mathrm{mM}$ Tris (pH 7.5), $100 \mathrm{mM}$ $\mathrm{NaCl}$, and $5 \%$ glycerol (buffer $\mathrm{A}$ ), and the protein was eluted with buffer A and $200 \mathrm{mM}$ imidazole. Fractions of $\mathrm{His}_{6} \Delta \mathrm{NVps} 4 \mathrm{p}\left(\mathrm{His}_{6} \Delta \mathrm{NVps} 4 \mathrm{p}\right.$ Q216A) were supplemented with $5 \mathrm{mM}$ EDTA, concentrated to a final volume of $5 \mathrm{ml}$ using Amicon Centriprep, and loaded into a 26/60 HiLoad Superdex (Amersham Biosciences) gel-filtration column equilibrated with $20 \mathrm{mM}$ Tris ( $\mathrm{pH} 7.5), 100 \mathrm{mM}$ $\mathrm{KCl}$, and $5 \%$ glycerol (buffer C). $\mathrm{His}_{6} \mathrm{Vps}_{4} \mathrm{p}$ was purified as the truncated construct, but the Ni-chelating column was equilibrated with buffer A containing $300 \mathrm{mM}$ (instead of $100 \mathrm{mM}$ ) $\mathrm{NaCl}, \mathrm{His}_{6} \mathrm{Vps} 4 \mathrm{p}$ was supplemented with $5 \mathrm{mM}$ EDTA, concentrated, diluted with $20 \mathrm{mM}$ Tris $(\mathrm{pH} 7.5)$ and $5 \%$ glycerol (buffer $\mathrm{B})$, and loaded into a Q-Sepharose column (Amersham Biosciences) equilibrated with buffer $B$. The protein eluted at around $300 \mathrm{mM} \mathrm{KCl}$ in a gradient between $100 \mathrm{mM}$ and $500 \mathrm{mM}$ $\mathrm{KCl}$ in buffer $\mathrm{B}$, followed by gel filtration in buffer $\mathrm{C}$. $\mathrm{His}_{6} \Delta \mathrm{NVps} 4 \mathrm{p}$ was crystallized in a sitting-drop vapordiffusion experiment at a concentration of $6.25 \mathrm{mg} / \mathrm{ml}$ at $19{ }^{\circ} \mathrm{C}$ against a reservoir containing $0.1 \mathrm{M}$ 4-morpholineethanesulfonic acid ( $\mathrm{pH} 5.9-6.5$ ) and $0.8-1.9 \mathrm{M} \mathrm{MgSO}_{4}$. The crystallization drop contained $1 \mu \mathrm{l}$ of protein solution and $0.5 \mu 1$ of reservoir. Crystals grew within $2-5$ days. To freeze the crystals in propane, the reservoir buffer was mixed with D-(-)-2,3-butanediol (FLUKA) in a ratio of 17:3.

\section{Activity assays}

The ATPase activity of $1 \mu \mathrm{g}$ and $2 \mu \mathrm{g}$ of $\mathrm{His}_{6} \mathrm{Vps} 4 \mathrm{p}$ or $25 \mu \mathrm{g}$ of $\mathrm{His}_{6} \Delta \mathrm{NVps} 4 \mathrm{p}$ in $50 \mathrm{mM}$ Tris (pH 7.5), $100 \mathrm{mM}$ $\mathrm{NaCl}$, and $3 \%$ glycerol (buffer $\mathrm{D}$ ) was determined as reported earlier. ${ }^{40}$

\section{Gel filtration}

$\mathrm{His}_{6} \mathrm{Vps}_{4}$ p, $\mathrm{His}_{6} \Delta \mathrm{NVps} 4 \mathrm{p}$, and $\mathrm{His}_{6} \Delta \mathrm{NVps} 4 \mathrm{pQ} 216 \mathrm{~A}$ in buffer $C$ with a concentration of $5 \mathrm{mg} / \mathrm{ml}$ were loaded into a HiLoad S300 column equilibrated with buffer C. For molecular standards, RibA, cytochrome, ovalbumin, and bovine serum albumin were used.

\section{Data collection and structure determination}

Data were collected at the Swiss Light Source (Villigen, Switzerland) and processed in space group $P 6_{5} 22$ to a resolution of $3.35 \AA$ using the programs MOSFLM and SCALA. $^{28,40}$ The structure of $\mathrm{His}_{6} \Delta$ NVps4p was solved by molecular replacement methods using the program PHASER $^{28}$ and $V_{p s} 4 B^{8}$ as a search model. One clear solutions was found. Model building was carried out with the program MAIN2000 ${ }^{41}$ and subsequently refined using $\mathrm{CNS}^{42}$ and Refmac TLS-refinement ${ }^{28}$ with Engh and Huber parameters. In addition, model bias was reduced by calculating simulated annealing composite omit maps in CNS. ${ }^{42}$

\section{Cryo-EM}

After elution from the Ni-chelating column and dialysis against $20 \mathrm{mM}$ Tris (pH 7.5), $\mathrm{His}_{6} \mathrm{Vps}_{4} \mathrm{p}, \mathrm{His}_{6} \Delta \mathrm{NVps} 4 \mathrm{p}$, and $\mathrm{His}_{6} \Delta \mathrm{NVps} 4 \mathrm{pQ} 216 \mathrm{~A}$ were mixed with AMPPNP and EDTA to final concentrations of $1 \mathrm{mM}$ and $5 \mathrm{mM}$, respectively, applied to a holey carbon film from Quantifoil (Germany) at a concentration of about $2.5 \mathrm{mg} / \mathrm{ml}$, and frozen in liquid ethane. The frozen grids were analyzed on a Philips CM200FEG electron microscope operating at $200 \mathrm{kV}$. The images were recorded on Kodak SO 163 films at a magnification of $\times 50,000$ under low-dose conditions $\left(10 \mathrm{e}^{-} / \AA^{2}\right)$. The micrographs were digitized on a Heidelberg Primescan with a pixel size of $10 \mu \mathrm{m}$, corresponding to $2 \AA$ at the specimen level.

\section{Image analysis and refinement procedure}

A total of 6904 particles were carefully selected from micrographs with a defocus between $-3.0 \mu \mathrm{m}$ and $-5.0 \mu \mathrm{m}$, reference-free-aligned, and classified without imposed symmetry using the EMAN package. ${ }^{32}$ A clear 7-fold symmetry of the top views and a cylindrical double-ring structure of the side views were visible. A starting model was created with the SPIDER package ${ }^{43}$ containing 14 spheres arranged into two heptameric stacked rings with the dimensions and symmetry of the class averages of the side and top views. But due to low contrast in cryo-EM, both programs were unable to safely discriminate from the top and side views, possibly as a result of significant conformational variability of the Vps4p tetradecamer. Sophisticated image processing tools to assess such conformational variability are yet to be fully developed. ${ }^{33}$ A total of 2473 particles comprising equal amounts of top and side views, which aligned correctly on the projections of the starting model, were used for calculating a better starting model, followed by eight cycles of refinement with angular increments of $5^{\circ}$ and implied 7 -fold symmetry. Subsequently, three cycles of refinement with the contrast transfer function-corrected images were performed with the EMAN package. ${ }^{32}$ A final refinement that included 5999 of the 6904 particles was performed and converted into the same volume, but with a reduced resolution. The contrast transfer function was estimated using the ctffind and ctftilt programs ${ }^{44}$ and applied to the images with the SPIDER package. ${ }^{43}$ The $18-\AA$ resolution was calculated by the 0.5 Fourier shell correlation criterion with both the SPIDER $^{43}$ and the EMAN ${ }^{32}$ packages. The averaged rotational power spectra of Vps4p particles were performed with $\mathrm{XMIPP}^{31}$ and the contour plots of the sections through the median plane perpendicular to the $z$-axis of the Vps4p reconstruction were performed with the program Semper. ${ }^{45}$ The classification of experimental projections based on their rotational power spectra was performed with XMIPP. ${ }^{31}$

\section{Fitting of the His ${ }_{6} \Delta \mathrm{NVps} 4 \mathrm{p}$ dimer into the EM map}

The atomic structure of the $\mathrm{His}_{6} \Delta \mathrm{NVps} 4 \mathrm{p}$ dimer was manually fitted into the tetradecameric EM map with the program MAIN2000, ${ }^{41}$ avoiding clashes between the $C \alpha$ chains. The relative positions of the domains were further optimized using the model-rigid-body and model-minimize routines of CNS. ${ }^{42}$

In addition, with the programs MAIN2000 ${ }^{41}$ and CCP4, ${ }^{28}$ the two resulting heptameric ring models were separated and fitted into a head-to-tail fashion, with the closed ring as the head and with the open ring as the tail, and in a tail-totail configuration on the experimental EM map, with further consideration that the rings are close enough to interact with each other. All three models were refined, and correlation coefficients were calculated using Situs. ${ }^{34}$

\section{MD simulations}

The protonation state of the three different dimers was calculated, and missing short loops and residues from the 
C-terminus were inserted using the program WHAT IF. ${ }^{46}$ The dimers were solvated in water boxes using TIP $4 \mathrm{P}^{47}$ as a water model. The ionic strength was adjusted to $\sim 0.15 \mathrm{M}$ by adding $\mathrm{Na}^{+}$and $\mathrm{Cl}^{-}$. In total, the system size was $\sim 300,000$ atoms in each case. For the protein, the optimized potential for liquid simulations all-atom force field was used. ${ }^{48}$ The systems were energy-minimized, and the solvent was equilibrated for 300 ps by applying position restraints on the protein heavy atoms. Subsequently, trajectories $10 \mathrm{~ns}$ long were obtained by free MD simulations, using a preversion of the GROMACS 4.0 simulation software. ${ }^{49}$ Electrostatic interactions were calculated explicitly at a distance smaller than $1 \mathrm{~nm}$, and long-distance electrostatic interactions were calculated at every step by particle-mesh Ewald summation. ${ }^{50}$ Lennard-Jones interactions were calculated with a cutoff of $1 \mathrm{~nm}$. All bonds were constrained using the LINCS algorithm, ${ }^{51}$ and the integration time step was $2 \mathrm{fs}$. The simulation temperature was kept constant by weakly $(\tau=0.1 \mathrm{ps})$ coupling the protein and the solvent separately to a temperature bath of $300 \mathrm{~K} .^{52}$ Likewise, the pressure was kept constant by weakly coupling the system to a pressure of 1 bar.

\section{Protein Data Bank accession number}

The atomic coordinates and structure factors of $\mathrm{His}_{6} \Delta \mathrm{NVps} 4 \mathrm{p}$ have been deposited in the Protein Data Bank with accession number 2rko.

\section{Acknowledgements}

We acknowledge financial support from the Swiss NCCR Structural Biology to M.G.G and A.E. We thank the staff of beamline XOGSA of the Swiss Light Source for excellent technical assistance, Dr. M. Gregorini for the introduction of C.H. to the programs SPIDER and EMAN, Prof. T. Schirmer and Dr. H.W. Rémigy for helpful discussion, and Dr. A. Philippsen for helpful discussion and critical reading of the manuscript.

\section{Supplementary Data}

Supplementary data associated with this article can be found, in the online version, at doi:10.1016/ j.jmb.2008.01.010

\section{References}

1. Katzmann, D. J., Odorizzi, G. \& Emr, S. D. (2002). Receptor downregulation and multivesicular-body sorting. Nat. Rev. Mol. Cell Biol. 3, 893-905.

2. Thompson, B. J., Mathieu, J., Sung, H. H., Loeser, E., Roth, P. \& Cohen, S. M. (2006). Tumor suppressor properties of the ESCRT-II complex component Vps25 in Drosophila. Dev. Cell, 9, 711-720.

3. Mahul-Mellier, A. L., Hemming, F. J., Blot, B., Fraboulet, S. \& Sadoul, R. (2006). Alix, making a link between apoptosis-linked gene-2, the endosomal sorting complexes required for transport, and neuronal death in vivo. J. Neurosci. 26, 542-549.
4. Pornillos, O., Higginson, D. S., Stray, K. M., Fisher, R. D., Garrus, J. E., Payne, M. et al. (2003). HIV gag mimics the Tsg101-recruiting activity of the human Hrs protein. J. Cell Biol. 162, 425-434.

5. Hurley, J. H. \& Emr, S. D. (2006). The ESCRT complexes: structure and mechanism of a membranetrafficking network. Annu. Rev. Biophys. Biomol. Struct. 35, 277-298.

6. Babst, M., Wendland, B., Estepa, E. J. \& Emr, S. D. (1998). The Vps4p AAA-ATPase regulates membrane association of a $\mathrm{Vps}$ protein complex required for normal endosome function. EMBO J. 17, 2982-2993.

7. Neuwald, A. F., Aravind, J. L., Spouge, J. L. \& Koonin, E. V. (1999). $\mathrm{AAA}^{+}$: a class of chaperone-like ATPases associated with the assembly, operation, and assembly of protein complexes. Genome Res. 9, 27-43.

8. Scott, A., Chung, H. Y., Gonciarz-Swiatek, M., Hill, G. C., Whitby, F. G., Gaspar, J. et al. (2005). Structural and mechanistic studies of VPS4 proteins. EMBO J. 24, 3658-3669.

9. Bochtler, M., Hartmann, C., Song, H. K., Bourenkov, G. P., Bartunik, H. D. \& Huber, R. (2000). The structures of HslU and the ATP-dependent protease HslUHslV. Nature, 403, 800-805.

10. Bieniossek, C., Schalch, T., Bumann, M., Meister, M., Meier, R. \& Baumann, U. (2006). The molecular architecture of the metalloprotease FtsH. Proc. Natl Acad. Sci. USA, 103, 3066-3071.

11. Scott, A., Gaspar, J., Stuchell-Brereton, M. D., Alam, S. L., Skalicky, J. J. \& Sundquist, W. I. (2005). Structure and ESCRT-III protein interactions of the MIT domain of human Vps4A. Proc. Natl Acad. Sci. USA, 102, 13813-13818.

12. Takasu, H., Jee, J. G., Ohno, A., Goda, N., Fujiwara, K., Tochio, H. et al. (2005). Structural characterization of the MIT domain from human Vps4B. Biochem. Biophys. Res. Commun. 334, 460-465.

13. Stuchell-Brereton, M. D., Skalicky, J. J., Kieffer, C., Karren, M. A., Ghaffarian, S. \& Sundquist, W. I. (2007). ESCRT-III recognition by VPS4 ATPases. Nature, 449, 740-744.

14. Obita, T., Sakasena, S., Ghazi-Tabatabai, S., Gill, D. J., Perisic, O., Emr, S. D. \& Williams, R. L. (2007). Structural basis for selective recognition of ESCRT-III by the AAA ATPase Vps4. Nature, 449, 735-739.

15. Xiao, J., Xia, H., Yoshino-Koh, K., Zhou, J. \& Xu, Z. (2007). Structural characterization of the ATPase reaction cycle of endosomal AAA protein VpsJ. Mol. Biol. 374, 655-670.

16. DeLaBarre, B. \& Brunger, A. T. (2003). Complete structure of $\mathrm{p} 97 /$ valosin-containing protein reveals communication between nucleotide domains. Nat. Struct. Biol. Mol. Biol. 10, 856-863.

17. Vajjhala, P. R., Wong, J. S., To, H. Y. \& Munn, A. L. (2006). The beta domain is required for Vps4p oligomerization into a functional active ATPase. FEBS J. 273, 2357-2373.

18. Azmi, I., Davies, B., Dimaano, C., Payne, J., Eckert, D., Babst, M. \& Katzmann, D. J. (2006). Recycling of ESCRTs by the AAA-ATPase Vps4 is regulated by a conserved VSL region in Vta1. J. Cell Biol. 172, 705-717.

19. Lottridge, J. M., Flannery, A. R., Vincelli, L. J. \& Stevens, T. H. (2006). Vta1p and Vps46p regulate the membrane association and ATPase activity of Vps4p at the yeast multivesicular body. Proc. Natl Acad. Sci. USA, 103, 6202-6207.

20. Vajjhala, P. R., Catchpoole, E., Nguyen, C. H., Kistler, C. \& Munn, A. L. (2007). Vps4 regulates a subset of 
protein interactions at the multivesicular endosome. FEBS J. 274, 1894-1907.

21. Shim, S., Kimpler, L. A. \& Hanson, P. I. (2007). Structure/function analysis of four core ESCRT-III proteins reveals common regulatory role for extreme C-terminal domain. Traffic, 8, 1068-1079.

22. Yeo, S. C. L., Xu, L., Ren, J., Boulton, V. J., Wagle, M. D., Liu, C. et al. (2003). Vps20p and Vta1p interact with Vps4p and function in multivesicular body sorting and endosomal transport in Saccharomyces cerevisiae. I. Cell Sci. 116, 3957-3970.

23. Nickerson, D. P., West, M. \& Odorizzi, G. (2006). Did2 coordinates Vps4-mediated dissociation of ESCRT-III from endosomes. J. Cell Biol. 175, 715-720.

24. DeLaBarre, B. \& Brunger, A. T. (2005). Nucleotide dependent motion and mechanism of the action of p97/VCP. J. Mol. Biol. 347, 437-452.

25. Huyton, T., Pye, V. E., Briggs, L. C., Flynn, T. C., Beuron, F., Kondo, H. et al. (2003). The crystal structure of murine p97/VCP at 3.6 A. J. Struct. Biol. 144, 337-348.

26. Guo, A., Maurizi, M. R., Esser, L. \& Xia, D. (2002). Crystal structure of $\mathrm{ClpA}$, an Hsp100 chaperone and regulator of ClpAP protease. J. Biol. Chem. 277, 46743-46752.

27. Lee, S., Sowa, M. E., Watanabe, Y., Sigler, P. B., Chiu, W., Yoshida, M. \& Tsai, F. (2003). The structure of ClpB: a molecular chaperone that rescues proteins from an aggregated state. Cell, 115, 229-240.

28. Collaborative Computational Project, Number 4. (1994). The CCP4 suite: program for protein crystallography. Acta Crystallogr. Sect. D, 50, 760-763.

29. Krissinel, E. \& Hendrick, K. (2005). Detection of protein assemblies in crystals. In CompLife LNBI 3695 (Berthold, M. R., ed), pp. 163-174, Springer-Verlag, Heidelberg.

30. Penczek, P., Radermacher, M. \& Frank, J. (1992). Three-dimensional reconstruction of single particles embedded in ice. Ultramicroscopy, 40, 33-53.

31. Sorzano, C. O. S., Marabini, R., Velazquez-Muriel, J., Bilbao-Castro, J. R., Scheres, S. H. W., Carazo, J. M. \& Pascual-Montano, A. (2004). XMIPP: a new generation of an open-source image processing package for electron microscopy. J. Struct. Biol. 148, 194-204.

32. Ludtke, S. J., Baldwin, P. R. \& Chiu, W. (1999). EMAN: semiautomated software for high-resolution singleparticle reconstructions. J. Struct. Biol. 128, 82-97.

33. Leschziner, A. E. \& Nogales, E. (2007). Visualization flexibility at molecular resolution: analysis of heterogeneity in single-particle electron microscopy reconstructions. Annu. Rev. Biophys. Biomol. Struct. 36 43-62.

34. Wriggers, W., Milligan, R. A. \& McCammon, J. A (1999). Situs: a package for docking crystal structures into low-resolution maps from electron microscopy. J. Struct. Biol. 125, 185-195.

35. Scheuring, S., Röhricht, R. A., Schöning-Burkhardt, B. Beyer, A., Müller, S., Abts, H. F. \& Köhrer, K. (2001) Mammalian cells express two VPS4 proteins both of which are involved in intracellular protein trafficking. J. Mol. Biol. 312, 469-480.

36. Akoev, V., Gogol, E. P., Barnett, M. E. \& Zolkiewski, M. (2004). Nucleotide-induced switch in oligomerization of the AAA ${ }^{+}$ATPase ClpB. Protein Sci. 13, 567-574.
37. Rohrwild, M., Pfeifer, G., Santarius, U., Müller, S. A., Huang, H. C., Engel, A. et al. (1997). The ATPdependent HslVU protease from Escherichia coli is a four-ring structure resembling the proteasome. Nat. Struct. Biol. Mol. Biol. 4, 133-139.

38. Zhang, X., Stoffels, K., Wurzbacher, S., Schoofs, G., Pfeifer, G., Banerjee, T. et al. (2004). The N-terminal coiled coil of the Rhodococcus erythropolis ARC AAAATPase is neither necessary for oligomerization nor nucleotide hydrolysis. J. Struct. Biol. 146, 155-165.

39. Rouiller, I., DeLaBarre, B., May, A. P., Weis, W. I., Brunger, A. T., Milligan, R. A. \& Wilson-Kubalek, E. M. (2002). Conformational changes of the multifunction p97 AAA ATPase during ATPase cycle. Nat. Struct. Biol. Mol. Biol. 9, 950-957.

40. Leslie, A. G. W. (1992). Recent changes to MOSFLM package for processing film and image plate data. CCP4 ESF-EACMB Newosl. Protein, 26.

41. Turk, D. (1992). Weiterentwicklung eines Programms für Molekülgraphik und Elektronendichte-Manipulation und seine Anwendung auf verschiedene ProteinStrukturaufklärungen. Ph.D. Thesis, Technische Univ. München, Munich.

42. Brunger, A. T., Adams, P. D., Clore, G. M., DeLano, W. L., Gros, P., Grosse-Kunstleve, R. W. et al. (1998). Crystallography, and NMR system: a new software suite for macromolecular structure determination. Acta Crystallogr. Sect. D, 54, 905-921.

43. Frank, J. \& Rademacher, M. (1996). SPIDER and WEB: processing and visualization of images in 3D EM and related fields. J. Struct. Biol. 116, 190-199.

44. Mindell, J. A. \& Grigorieff, N. (2003). Accurate determination of local defocus and specimen tilt in electron microscopy. J. Struct. Biol. 142, 334-347.

45. Saxton, W. O. (1996). Semper: distortion compensation, selective averaging, 3-D reconstruction, and transfer function correction in a highly programmable system. J. Struct. Biol. 116, 230-236.

46. Vriend, G. (1990). WHAT IF-a molecular modeling and drug design program. J. Mol. Graphics, 8, 52-56.

47. Jorgensen, W. L., Chandrasekhar, J., Madura, J. D., Impey, R. W. \& Klein, M. L. (1983). Comparison of simple potential functions for simulating liquid water. J. Chem. Phys. 79, 926-935.

48. Jorgensen, W. L., Maxwell, D. S. \& Tirado-Rives, J. (1996). Development and testing of the OPLS-AA force field on conformational energetics and properties of organic liquids. J. Am. Chem. Soc. 118, 11225-11236.

49. van der Spoel, D., Lindahl, E., Hess, B., Groenhof, G., Mark, A. E. \& Berendsen, H. J. C. (2005). GROMACS: fast, flexible, and free. J. Comput. Chem. 26, 1701-1718.

50. Darden, T., York, D. \& Pedersen, L. (1993). Particle mesh Ewald-an $N \log (N)$ method for Ewald sums in large systems. J. Chem. Phys. 98, 10089-10092.

51. Hess, B., Bekker, H., Berendsen, H. J. C. \& Fraaije, J. G. E. M. (1997). LINCS: a linear constraint solver for molecular simulations. J. Comput. Chem. 18, 1463-1472.

52. Berendsen, H. J. C., Postma, J. P. M., van Gunsteren, W. F., Di Nola, A. \& Haak, J. R. (1984). Molecular dynamics with coupling to an external bath. J. Chem. Phys. 81, 3684-3690. 